TRANSACTIONS OF THE

AMERICAN MATHEMATICAL SOCIETY

Volume 364, Number 6, June 2012, Pages 2933-2958

S 0002-9947(2012)05381-5

Article electronically published on February 14, 2012

\title{
SUM-INTEGRAL INTERPOLATORS AND THE EULER-MACLAURIN FORMULA FOR POLYTOPES
}

\author{
STAVROS GAROUFALIDIS AND JAMES POMMERSHEIM
}

\begin{abstract}
A local lattice point counting formula, and more generally a local Euler-Maclaurin formula follow by comparing two natural families of meromorphic functions on the dual of a rational vector space $V$, namely the family of exponential sums $(S)$ and the family of exponential integrals $(I)$ parametrized by the set of rational polytopes in $V$. The paper introduces the notion of an interpolator between these two families of meromorphic functions. We prove that every rigid complement map in $V$ gives rise to an effectively computable SI-interpolator (and a local Euler-Maclaurin formula), an IS-interpolator (and a reverse local Euler-Maclaurin formula) and an IS-interpolator (which interpolates between integrals and sums over interior lattice points). Rigid complement maps can be constructed by choosing an inner product on $V$ or by choosing a complete flag in $V$. The corresponding interpolators generalize and unify the work of Berline-Vergne, Pommersheim-Thomas, and Morelli.
\end{abstract}

\section{Contents}

1. Introduction

1.1. What is a local lattice-point counting formula?

1.2. What is a local Euler-Maclaurin summation formula?

1.3. An informal presentation of the results of this paper

2. Polytopes, exponential sums/integrals and interpolators

2.1. Polytopes

2.2. Exponential sums and integrals

2.3. SI-interpolators

2.4. Rigid complement maps

3. Statement of the results

3.1. SI-interpolators and local Euler-Maclaurin formula 2942

3.2. IS-interpolators and reverse Euler-Maclaurin formula 2944

3.3. IS $^{0}$-interpolators and Morelli's work 2945

4. Proofs

4.1. Proof of Theorem 1

4.2. Proof of Theorem 2

4.3. Proof of Theorem 4

4.4. Proof of Theorems 5 and 7

5. Computations and examples

5.1. Computation of $\mu^{\Psi}$ for low-dimensional cones 2949

5.2. Examples

Received by the editors February 18, 2010 and, in revised form, May 20, 2010. 2010 Mathematics Subject Classification. Primary 57N10; Secondary 57M25.

Key words and phrases. Polytopes, Euler-Maclaurin formula, reverse Euler-Maclaurin formula, lattice points, flag varieties, exponential sums, exponential integrals, interpolators.

The first author was supported in part by NSF. 
6. Comparison with Morelli's work

6.1. Computation of $\nu^{\Psi}$ for low-dimensional cones 2956

6.2. Morelli's coincidence in dimensions 1 and $2 \quad 2957$

References

\section{INTRODUCTION}

1.1. What is a local lattice-point counting formula? The relationship between sums and integrals has been of interest to mathematicians since the ancient Greeks. The classical Euler-Maclaurin formula, discovered in the first half of the eighteenth century shortly after the development of modern calculus, may be viewed as a relationship between the sum of a function over the lattice points in a 1-dimensional polytope with integer vertices and the integral of the function over the polytope. One naturally asks the same question in higher dimensions: Given a polytope $P$ in an $n$-dimensional space $V$ equipped with an $n$-dimensional lattice $\Lambda$ and a function $f$ on $V$, can one express the sum of $f$ over the lattice points in $P$ in terms of the integral $f$ over $P$ ? In such a formula, one would expect a main term involving the integral of $f$ over $P$ as well as correction terms involving the integrals of $f$ over the proper faces $F \subset P$.

In the simplest case, let us suppose that $f$ is a constant function. Then, the question becomes that of expressing the number of lattice points in $P$ in terms of the volume of $P$ and the volumes of the faces $F$ of $P$. If $P$ is 2-dimensional, the celebrated Pick's formula Pi]

$$
\#(P)=A+\frac{1}{2} b+1
$$

expresses the number $\#(P)$ of lattice points in a convex lattice polygon in terms of its area $A=\operatorname{vol}(P)$, and the number of lattice points $b=\operatorname{vol}(\partial P)$ of its boundary. For example, we have:

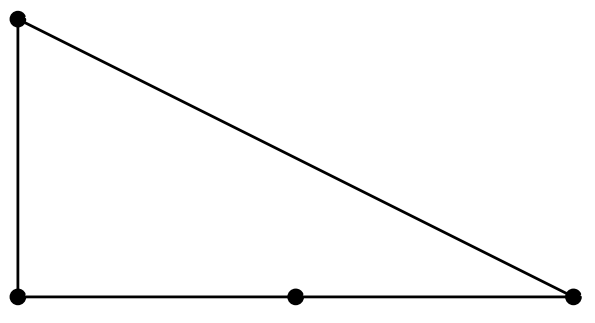

$$
\#(P)=4, A=1, b=4 .
$$

Figure 1

Here and throughout, we follow the usual convention that all volumes $\operatorname{vol}(F)$ of faces are normalized so that a lattice basis of $\Lambda \cap L$, where $L$ is the linear space parallel to $F$, has volume 1. Unfortunately, Pick's formula is not local, due to the presence of the term 1 . Here, locality means that for each face $F$ of $P$, the formula contains a term that is the volume $\operatorname{vol}(F)$ multiplied by a coefficient that depends only on the supporting cone $\operatorname{Supp}(P, F)$ to $P$ along $F$. The supporting cone is defined as the union of rays whose endpoints are in $F$ and which remain in $P$ for a positive distance. See Section 2.1 for a precise definition. McMullen $\mathrm{McM}$ ] proved 
the existence of local lattice-point counting formulas. More precisely, he proved the existence of a function $\mu$ from rational, convex cones to rational numbers such that for any integral polytope $P$, the number $\#(P)$ of lattice points in $P$ is given by

$$
\#(P)=\sum_{F} \mu(\operatorname{Supp}(P, F)) \operatorname{vol}(F) \text {. }
$$

Here the sum is taken over all faces $F$ of $P$, and $\operatorname{vol}(P)$ denotes the volume of the face $F$.

Part of the difficulty constructing and computing a function $\mu$ that satisfies (11) is that $\mu$ is far from unique. The second author and Thomas gave an explicit construction of a rational-valued function $\mu$ satisfying (1), given a fixed complement map, a notion introduced in Thomas's thesis (cf. [Th]). A complement map is a systematic choice of complements of linear subspaces of a vector space; see Section 2.4 for a precise definition. Also note that all of the complement maps in this paper will be rigid. Two natural ways to get a rigid complement map are to choose: (1) an inner product, or (2) a complete flag. Given an inner product or a complete flag, [PT] effectively construct a map $\mu$ that satisfies equation (11). For the triangle depicted in Figure 1, the Pommersheim-Thomas values of $\mu$ at the supporting cones to the vertices for an inner product or complete flags are as follows:
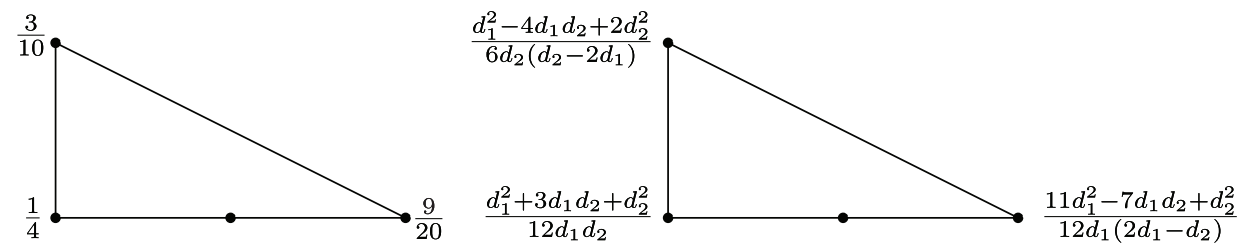

Figure 2

The values on the left arise from the standard inner product on $\mathbb{Z}^{2}$, according to [PT, Cor.1], and may be computed by multiplying out the Todd polynomial in the ring presentation given in $[\mathrm{PT}$, Prop.2]. Readers wishing more details on this should consult Section 5.2. The values in the figure on the right are those arising from the choice of the complete flag in $V^{*}=\mathbb{Z}^{2}$ whose 1-dimensional subspace is spanned by the point $\left(d_{1}, d_{2}\right)$. These values can be computed using the method outlined in $\mathrm{Mo}$, p.198]. They can also be computed by multiplying out the Todd polynomials using [PT, Thm.3]. One feature of this construction is that if one chooses a complement map arising from a complete flag, one recovers exactly the lattice point formulas of Morelli $\mathrm{Mo}$, who gave a function $\mu$ that satisfies equation (11) and takes values in the field of rational functions on a Grassmannian. For example, in the triangle on the right in Figure 2, the values shown are rational functions on the Grassmannian of 1-dimensional subspaces of $V^{*}$. The construction of $[\mathrm{PT}]$ is based on the theory of toric varieties and gives an answer to a question of Danilov about the existence of a local expression for the Todd class of a toric variety.

1.2. What is a local Euler-Maclaurin summation formula? Returning to the Euler-Maclaurin question, Berline and Vergne constructed in BV an explicit local Euler-Maclaurin formula for the sum of a polynomial function $f$ over the lattice points $P \cap \Lambda$ of an $n$-dimensional polytope $P$ in a rational vector space $V$ 
with lattice $\Lambda$. The Berline-Vergne formula has the form

$$
\sum_{x \in P \cap \Lambda} f(x)=\sum_{F} \int_{F} D(P, F) \cdot f
$$

where the sum is over the set of faces $F$ of $P$, and $D(P, F)$ an infinite-order constantcoefficient differential operator $D(P, F)$ that depends only on the supporting cone $\operatorname{Supp}(P, F)$. Equation (2) is a generalization of (11). Indeed, if $D(P, F)$ satisfies (2), and we define $\mu(\operatorname{Supp}(P, F))$ to be the constant term of $D(P, F)$, then the local lattice-point formula (10) holds.

As in McMullen's case, the infinite order differential operators $D(P, F)$ are not uniquely determined by (2). The construction of Berline-Vergne requires an inner product on the vector space $V$, and their results apply to rational polytopes, rather than just integral polytopes. Their construction associates to each cone $K$ in $V$ a meromorphic function $\mu(K)$ on the dual space that is regular at the origin. For cones of dimension at most 2 , the value of this function at 0 (the constant term of the operator $D(P, F)$ ) coincides with the $\mu$ constructed in [PT] for the special case of complement maps arising from an inner product. In this way, for the example triangle in Figure 2, the Berline-Vergne construction recovers the the numbers $\frac{1}{4}, \frac{9}{20}, \frac{3}{10}$ arising out of the inner product case of the Pommersheim-Thomas construction. The fact that this coincidence holds in general is new to this paper; see Theorem 1 properties (5) and (6).

1.3. An informal presentation of the results of this paper. The local EulerMaclaurin formula (2) is a consequence of a relationship between the integral and the sum of an exponential function over a polytope. In this paper, we introduce the concept of an interpolator between the families of exponential sums $(S)$ and exponential integrals $(I)$ over rational polytopes in a rational vector space $V$. We now discuss our results about interpolators informally, leaving the precise definitions and statements for Sections 2 and 3 .

If $P$ is a rational polytope, or more generally a rational polyhedron (region, not necessarily compact, defined by linear inequalities with rational coefficients) in a rational vector space $V$, one can associate to $P$ two important meromorphic functions, the exponential sum $S(P) \in \mathcal{M}\left(V^{*}\right)$ and the exponential integral $I(P) \in$ $\mathcal{M}\left(V^{*}\right)$, where $\mathcal{M}\left(V^{*}\right)$ is the algebra of meromorphic functions on the dual space $V^{*} \otimes \mathbb{C}$. The values of these functions at a point $\xi \in V^{*} \otimes \mathbb{C}$ are given by:

$$
S(P)(\xi)=\sum_{x \in P \cap \Lambda} e^{\langle\xi, x\rangle}, \quad I(P)(\xi)=\int_{P} e^{\langle\xi, x\rangle} d x
$$

provided $\left|e^{\langle\xi, x\rangle}\right|$ is summable (resp. integrable) over $P$. Here, the integral is taken with respect to the relative Lebesgue measure on aff $(P)$, normalized so that a basis of $\Lambda \cap W$, where $W$ is the linear subspace parallel to the affine span of $P$, has volume 1. The fact that equations (3) define meromorphic functions, as well as the precise characterization and properties of the functions $S$ and $I$, is essentially the content of Lawrence's theorem La, reviewed in Section 2.2

The local Euler-Maclaurin formula (2) follows in a straightforward manner from a formula of the following shape for a rational polyhedron $P$ :

$$
S(P)=\sum_{F} \mu(\operatorname{Supp}(P, F)) I(F),
$$


where $\mu$ is a function on the set of cones in $V$ with values in $\mathcal{M}\left(V^{*}\right)$. We call such a function an SI-interpolator, or simply an interpolator. The main result of this paper (Theorem 11) states that a complement map on the vector space $V$ gives rise in a natural way to an effectively computable SI-interpolator on $V$, and hence a local Euler-Maclaurin formula of the form (2); see Theorem 2.

Thus, in particular, an inner product on $V$ or a complete flag in $V$ gives rise to a local Euler-Maclaurin formula. These interpolators have interesting connections with previous results. For complement maps arising from the choice of an inner product, one recovers the $\mu$ constructed in [BV]. In addition, we show that the values of these functions $\mu$ at 0 are given by the functions $\mu$ in $[\mathrm{PT}$ for cones of dimension at most 2 and conjecture that these coincidences hold in all dimensions. In the case of complete flags, one obtains interpolators $\mu$ that are entirely new, as can be seen by calculations in dimension 2 . In this case, the values of the constant term $\mu(K)(0)$ coincide with values constructed by Morelli for cones of dimension at most 2 , and conjecturally in all dimensions. One can also vary the chosen flag, thus associating to each cone $K$ a meromorphic function $\mu(K)$ on $\operatorname{Fl}\left(V^{*}\right) \times V^{*}$, where $\operatorname{Fl}\left(V^{*}\right)$ is the complete flag variety of $V$. This function $\mu(K)$ is naturally defined and effectively computable independent of any choices; see Theorem 3 . In Morelli's work, he constructs what amounts to the constant term $\mu(K)(0)$ of this meromorphic function. Working dimension-by-dimension, Morelli chooses to view this term as a rational function on a Grassmannian, rather than on the entire flag variety.

In Section 3.2 we show that a complement map on $V$ also leads naturally to the construction of a local IS-interpolator, expressing the exponential integral $I(P)$ in terms of the exponential sums $S(F)$, for $F$ a face of $P$; see Theorem 4 . This construction, which works only in the case of integral polyhedra, allows one to obtain a reverse local Euler-Maclaurin formula expressing the integral of a polynomial function over a polytope in terms of the sums of the function over lattice points in the various faces of the polytope; see Theorem 6 .

Finally, we show how a complement map yields an $\mathrm{IS}^{0}$-interpolator, where we use $\mathrm{S}^{0}$ to denote the sum over interior lattice points of a polytope. Part of the interest in $\mathrm{IS}^{0}$-interpolators lies in an observation of Morelli, who asked essentially (in the language of the present paper) if the same function can simultaneously serve as the constant term of an SI-interpolator and as the constant term of an IS $^{0}$-interpolator on the dual space. He observed that the constant terms that he constructs do exactly this in dimensions at most 4 . We give a proof of these coincidences in dimensions 1 and 2 and extend these results to the case of complement maps arising from inner products. Our arguments are essentially geometric, in contrast to Morelli's rather algebraic argument. (See Sections 3.3 and 6 for a complete discussion.)

Section 5 contains explicit computations of the functions $\mu(K)$ for some cones $K$ of dimensions at most 2 . We compute these functions for complement maps arising both from inner products and from complete flags. In Section 5.2, we exhibit equation (44) in the case of two triangles, including the triangle depicted in Figure 2 for both the inner product and complete flag cases, matching the constant terms with those constructed by Pommersheim-Thomas and Morelli shown in Figure 2. 

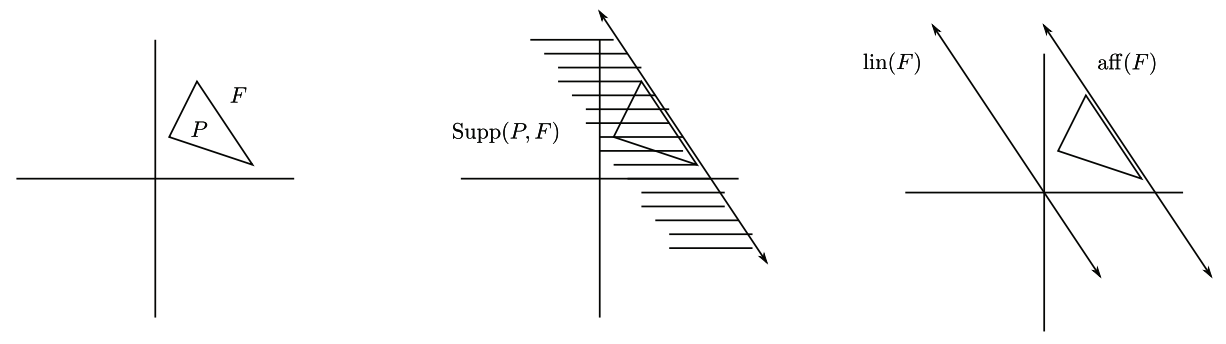

FiguRE 3

\section{Polytopes, exponential sums/integrals and interpolators}

2.1. Polytopes. We will use standard terminology for rational polytopes, polyhe$\mathrm{dra}$, cones and faces, following for example $\mathrm{Fu}$. We will fix a finite-dimensional lattice $\Lambda$, isomorphic to $\mathbb{Z}^{k}$ for some $k \in \mathbb{N}$, and consider the rational vector space $V=\Lambda \otimes_{\mathbb{Z}} \mathbb{Q}$. All polyhedra will be rational and convex, and all cones will be rational, polyhedral and may be affine. A polytope is a compact polyhedron. If $P$ is a polyhedron and $F$ is a face of $P$, then the tangent cone $\operatorname{Tan}(P, F)$ is defined by picking a point $x$ in the relative interior of $F$ and looking at all the directions that one can go and stay in $P$ :

$$
\operatorname{Tan}(P, F)=\{v \in V \mid x+\epsilon v \in P \text { for small } \epsilon>0\} .
$$

This cone is independent of the choice of $x$ and contains the origin. Let aff $(F)$ denote the affine span of $F$, i.e., the smallest affine subspace of $V$ that contains $F$. Let $\operatorname{lin}(F)$ denote the linear subspace of $V$ parallel to $\operatorname{aff}(F) \cdot \operatorname{lin}(F)$ is the maximal linear subspace of $V$ contained in $\operatorname{Tan}(P, F)$. The supporting cone $\operatorname{Supp}(P, F)$ is the tangent cone translated back to its original position:

$$
\operatorname{Supp}(P, F)=\operatorname{Tan}(P, F)+x
$$

for $x$ in the relative interior of $F$.

2.2. Exponential sums and integrals. Let $V^{*}=\operatorname{Hom}_{\mathbb{Q}}(V, \mathbb{Q})$ denote the dual vector space to $V$, and $\langle\cdot, \cdot\rangle: V^{*} \times V \longrightarrow \mathbb{Q}$ denote the natural evaluation pairing on $V$. By a meromorphic function on $V^{*}$ we will mean a meromorphic function on the complexified dual space $V_{\mathbb{C}}^{*}$, where $V_{\mathbb{C}}=V \otimes_{\mathbb{Q}} \mathbb{C}$ denotes the complexification of $V$. Let $\mathcal{M}\left(V^{*}\right)$ denote the algebra of meromorphic functions on $V^{*}$ and $\mathcal{M}^{r}\left(V^{*}\right)$ denote the subalgebra of meromorphic functions regular (i.e., analytic) at the origin. Note that if $W$ is a rational subspace of $V$, then $\Lambda^{\prime}=\Lambda \cap W$ is a full rank lattice in $W$ which naturally induces a relative Lebesgue measure on $W$, normalized so that a basis of $\Lambda^{\prime}$ has volume 1. Likewise, every rational affine subspace of $V$ has a natural measure induced by the lattice.

Given a polyhedron $P$ in a rational vector space $V$ with lattice $\Lambda$ as above, there are two important meromorphic functions $S(P)$ and $I(P)$ with rational coefficients in $V^{*}$, namely the generating function of exponential sums and exponential integrals.

These functions are uniquely characterized by the following properties.

(A1) If $P$ contains a straight line, then $S(P)=I(P)=0$.

(A2) $S$ (resp. $I$ ) is a valuation (resp. a simple (or solid) valuation). That is, if the characteristic functions of a family of polyhedra satisfy a relation 
$\sum_{i} r_{i} \chi\left(P_{i}\right)=0$, then the functions $S\left(P_{i}\right)$ satisfy the relation $\sum_{i} r_{i} S\left(P_{i}\right)=0$ (resp. restrict the sum to those $P_{i}$ that do not lie in a proper affine subspace of $V)$.

(A3) For every $s \in V$ and every $\xi \in V^{*}$ we have

$$
I(s+P)(\xi)=e^{\langle\xi, s\rangle} I(P)(\xi)
$$

and

$$
S(s+P)(\xi)=e^{\langle\xi, s\rangle} S(P)(\xi), \quad s \in \Lambda .
$$

(A4) If $\xi \in V^{*}$ is such that $\left|e^{\langle\xi, x\rangle}\right|$ is integrable (resp. absolutely summable) over $P$, then

$$
I(P)(\xi)=\int_{P} e^{\langle\xi, x\rangle} d m_{P}(x), \quad S(P)(\xi)=\sum_{x \in P \cap \Lambda} e^{\langle\xi, x\rangle},
$$

where $d m_{P}$ denotes the relative Lebesgue measure on aff $(P)$.

The existence of the functions $I$ and $S$ is by no means obvious and is essentially the content of Lawrence's theorem [La]. (A1)-(A4) determine the value of $I$ and $S$ on a simplicial cone as follows.

(A5) If $K=\operatorname{Cone}\left(v_{1}, \ldots, v_{k}\right)$ is a simplicial cone generated by $k$ independent vectors $v_{1}, \ldots, v_{k}$ (where $k=\operatorname{dim}(V)$ in the case of $S$ ) and $\square\left(v_{1}, \ldots, v_{k}\right.$ ) denotes the parallelepiped generated by $v_{1}, \ldots, v_{k}$, we have:

$$
\begin{gathered}
I(K)(\xi)=(-1)^{k} \frac{\operatorname{vol}\left(\square\left(v_{1}, \ldots, v_{k}\right)\right)}{\prod_{i=1}^{k}\left\langle\xi, v_{i}\right\rangle}, \\
S(K)(\xi)=\left(\sum_{x \in \square\left(v_{1}, \ldots, v_{k}\right) \cap \Lambda} e^{\langle\xi, x\rangle}\right) \prod_{i=1}^{k} \frac{1}{1-e^{\left\langle\xi, v_{i}\right\rangle}} .
\end{gathered}
$$

Since any polyhedron can be subdivided (or virtually subdivided, using a linear combination of characteristic functions) into a union of simplicial cones, together with equation (8), one obtains an algorithm to compute $I(P)$ and $S(P)$. The complexity of such an algorithm is discussed in $[\mathrm{Bv}$. The next theorem which computes $I(P)$ and $S(P)$ of a polyhedron in terms of the tangent cones of its vertices was obtained by Brion (using toric varieties) and Lawrence (using combinatorics) independently.

Proposition $2.1(\overline{\mathrm{Br}}, \mathrm{La}])$. Let $P$ be a polyhedron in $V$. Then,

$$
I(P)=\sum_{v \in \mathcal{V}(P)} I(\operatorname{Supp}(P, v)), \quad S(P)=\sum_{v \in \mathcal{V}(P)} S(\operatorname{Supp}(P, v)),
$$

where the sums are over the set $\mathcal{V}(P)$ of vertices of $P$.

2.3. SI-interpolators. So far, our discussion of the meromorphic functions $S(P)$ and $I(P)$ was parallel but independent: our formulas did not mix $S$ and $I$. It is natural to ask for an interpolation between $S(P)$ and $I(P)$. Indeed, if $P$ is a polytope, then letting $\xi=0$ reduces to a classic problem: expressing the number of lattice points in $P$ in terms of the volumes of the faces of $P$. Let $\mathcal{C}(V)$ denote the set of cones of $V$. 
Definition 2.2. An SI-interpolator (or simply, interpolator) on $V$ is a map

$$
\mu: \mathcal{C}(V) \longrightarrow \mathcal{M}\left(V^{*}\right)
$$

such that for any rational polyhedron $P$ in $V$ we have

$$
S(P)=\sum_{F \in \mathcal{F}(P)} \mu(\operatorname{Supp}(P, F)) I(F),
$$

where the sum is over the set $\mathcal{F}(P)$ of all faces of $P$. An interpolator is regular if $\mu$ takes values in $\mathcal{M}^{r}\left(V^{*}\right)$.

For the next definition, recall that if $W$ is a quotient of $V$ with projection $\pi: V \longrightarrow W$, then there is a natural map $\mathcal{C}(W) \longrightarrow \mathcal{C}(V)$ given by $K \mapsto$ $\pi^{-1}(K)$. Moreover, $W^{*}$ is naturally a subspace of $V^{*}$, and there is a restriction $\operatorname{map} \mathcal{M}\left(V^{*}\right) \longrightarrow \mathcal{M}\left(W^{*}\right)$.

Definition 2.3. An SI-interpolator $\mu$ on $V$ is hereditary if for every rational quotient $W$ of $V$, the composition $\bar{\mu}: \mathcal{C}(W) \longrightarrow \mathcal{M}\left(W^{*}\right)$ given by the following diagram:

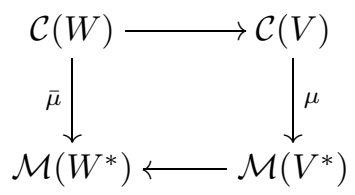

is an interpolator on $W$.

The next lemma reduces the checking of the interpolator equation (10) to the case of cones.

Lemma 2.4. $\mu$ is an interpolator if and only if it satisfies (10) for all cones $P$ in $V$.

Proof. This follows from Proposition 2.1. Indeed, we have

$$
\begin{aligned}
S(P) & =\sum_{v \in \mathcal{V}(P)} S(\operatorname{Supp}(P, v)) \\
& =\sum_{v \in \mathcal{V}(P)} \sum_{F^{\prime} \in \mathcal{F}(\operatorname{Supp}(P, v))} \mu\left(\operatorname{Supp}\left(\operatorname{Supp}(P, v), F^{\prime}\right)\right) I\left(F^{\prime}\right) .
\end{aligned}
$$

Since every face of $\operatorname{Supp}\left(\operatorname{Supp}(P, v), F^{\prime}\right)$ is a face of $\operatorname{Supp}(P, F)$ for some face $F$ of $P$, it follows that

$$
S(P)=\sum_{F \in \mathcal{F}(P)} \mu(\operatorname{Supp}(P, F)) \sum_{v \in \mathcal{V}(F)} I(\operatorname{Supp}(F, v))=\sum_{F \in \mathcal{F}(P)} \mu(\operatorname{Supp}(P, F)) I(F) .
$$

The result follows.

2.4. Rigid complement maps. A rigid complement map gives us a systematic way to extend a function on a linear subspace of a vector space to the entire vector space. This notion was introduced in the thesis of Thomas [Th and used in [PT], where it is shown that a choice of complement map leads naturally to a local formula for the number of lattice points in a polytope and a local formula for the Todd class of a toric variety. In our paper, we will only use the notion of a rigid complement map. The curious reader may consult [PT, Th] for the definition of the general complement maps. 
Definition 2.5. A rigid complement map on $V^{*}$ is a map $\Psi$ from a collection $\mathcal{L}^{\Psi}$ of linear subspaces in $V^{*}$ to the set of linear subspaces of $V^{*}$ satisfying two properties:

(a) For every $U \in \mathcal{L}^{\Psi}, \Psi(U)$ is complementary to $U$; i.e., $\Psi(U) \cap U=\{0\}$ and $\Psi(U)+U=V^{*}$.

(b) If $U_{1} \subset U_{2} \subset V^{*}$ with $U_{1}, U_{2} \in \mathcal{L}^{\Psi}$, then $\Psi\left(U_{2}\right) \subset \Psi\left(U_{1}\right)$.

There are two easy ways to construct rigid complement maps: (a) an inner product $Q$ on $V^{*}$, and (b) a complete flag $L$ on $V^{*}$.

Lemma 2.6. (a) An inner product $Q$ on $V^{*}$ defines a complement map $\Psi^{Q}$ with domain $\mathcal{L}^{Q}$ the set of all subspaces of $V^{*}$ such that

$$
\Psi(U)=U^{\perp},
$$

where $U^{\perp}$ denotes the subspace of $V^{*}$ perpendicular to $U \subset V^{*}$ under the inner product $Q$.

(b) A complete flag $L=\left(L_{0}, \ldots, L_{n}\right)$ on $V^{*}$ satisfying $L_{0}=\{0\} \subset L_{1} \subset \cdots \subset$ $L_{n}=V^{*}$ defines a complement map on $V^{*}$ by

$$
\Psi^{L}(U)=L_{n-\operatorname{dim}(U)}
$$

for any linear subspace $U$ of $V^{*}$ which is complementary to $L_{n-\operatorname{dim}(U)}$. This complement map is only defined generically, i.e., for subspaces that meet the flag generically.

Our main theorem, Theorem 1, requires cones that are generic with respect to a rigid complement map $\Psi$. Let us define what generic means. Fix a rigid complement map $\Psi$ on $V^{*}$.

Definition 2.7. (a) A quotient $W$ of $V$ is $\Psi$-generic if $W^{*} \in \mathcal{L}^{\Psi}$, where $W^{*} \subset V^{*}$.

(b) A subspace $U$ of $V$ is $\Psi$-generic if $V / U$ is $\Psi$-generic.

(c) A cone $K$ of $V$ is $\Psi$-generic if $\operatorname{lin}(F)$ is $\Psi$-generic for every face $F$ of $K$.

(d) A polyhedron $P$ of $V$ is $\Psi$-generic if for every face $F$ of $P$, $\operatorname{Supp}(P, F)$ is $\Psi$-generic.

The next lemma states that the notion of a complement map on $V^{*}$ is hereditary; i.e., a complement map on $V^{*}$ gives rise to a complement map on every $W^{*}$, where $W$ is a $\Psi$-generic rational quotient of $V$.

Lemma 2.8. If $\Psi$ is a rigid complement map on $V^{*}$ and if $W$ is a $\Psi$-generic rational quotient of $V$, then there is a natural complement map $\bar{\Psi}$ on $W^{*}$ defined by

$$
\bar{\Psi}(U)=\Psi(U) \cap W^{*}
$$

for all $U \subset W^{*}$ such that $\Psi(U) \cap W^{*}$ is complementary to $U$.

The next lemma gives the promised extension of functions on a linear subspace $W^{*}$ to ones on the entire space $V^{*}$.

Lemma 2.9. If $\Psi$ is a rigid complement map on $V^{*}$ and if $W$ is a $\Psi$-generic quotient of $V$, then the decomposition

$$
V^{*}=W^{*} \oplus \Psi\left(W^{*}\right)
$$

defines a unique linear projection map

$$
\pi: V^{*} \longrightarrow W^{*}
$$


that annihilates $\Psi\left(W^{*}\right)$ and is the identity on $W^{*}$. This allows any function on $W^{*}$ to be extended to $V^{*}$.

We now come to a main definition. Fix a rigid complement map $\Psi$ on $V^{*}$. Let $\mathcal{C}^{\Psi}(V)$ denote the set of $\Psi$-generic cones of $V$.

Definition 2.10. A $\Psi$-compatible interpolator $\mu$ on $V$ is a function $\mu: \mathcal{C}^{\Psi}(V) \longrightarrow$ $\mathcal{M}\left(V^{*}\right)$ satisfying:

(a) Equation (10) holds for all $\Psi$-generic polytopes $P$.

(b) $\mu$ is $\Psi$-hereditary, i.e., for every $\Psi$-generic quotient $W$ of $V$, the composition $\bar{\mu}: \mathcal{C}(W) \longrightarrow \mathcal{M}\left(W^{*}\right)$ given by the following diagram:

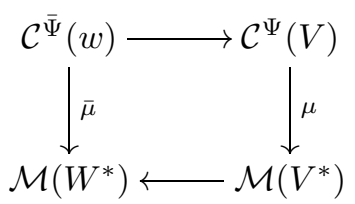

satisfies equation (10) for all $\bar{\Psi}$-generic polytopes $P$ in $W$.

(c) Moreover, the following diagram commutes:

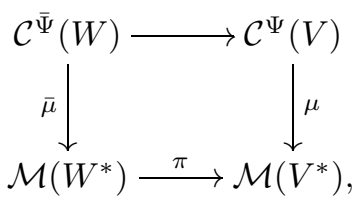

where $\pi$ is given in Lemma 2.9.

\section{Statement of the Results}

3.1. SI-interpolators and local Euler-Maclaurin formula. Our main result is that a rigid complement map $\Psi$ on $V^{*}$ determines algorithmically a unique $\Psi$ compatible interpolator $\mu^{\Psi}$ on $V$. This generalizes results of $[\mathrm{BV},[\mathrm{Mo}, \mathrm{PT}]$. The proof uses the techniques of [BV], which were a motivation and inspiration for us.

Theorem 1. (a) If $V$ is a rational vector space and $\Psi$ is a rigid complement map on $V^{*}$, then there is a unique $\Psi$-compatible interpolator $\mu^{\Psi}$.

(b) In addition, $\mu^{\Psi}$ satisfies the following properties on the collection of $\Psi$ generic cones on $V$ :

(1) (Additivity) If the characteristic functions of a finite collection of cones $K_{i}$ with vertex $v \in V$ satisfy the relation $\sum_{i} r_{i} \chi\left(K_{i}\right)=0$, then the functions $\mu^{\Psi}\left(K_{i}\right)$ satisfy the relation $\sum_{i} r_{i} \mu^{\Psi}\left(K_{i}\right)=0$.

(2) (Lattice invariance) If $v \in \Lambda$, then $\mu^{\Psi}(v+K)=\mu^{\Psi}(K)$.

(3) (Isometry equivariance) If $g$ is a lattice-preserving linear automorphism of $V$ and if $g^{*}$ is the inverse transpose, then $\mu^{g \Psi}(g(K))\left(g^{*}(\xi)\right)=\mu^{\Psi}(K)(\xi)$.

(4) (Regularity) $\mu^{\Psi}$ is regular at $\xi=0$.

Moreover, $\mu^{\Psi}$ generalizes previous results of [Mo and $[\mathrm{BV}]$ :

(5) (Constant term) Let $K$ be a top-dimensional cone in $V$ with vertex at zero, and suppose that $V$ has dimension at most 2 . Then for $\Psi$ arising from an inner product or a complete flag, the constant term of $\mu^{\Psi}(K)$ agrees with the Todd class coefficient $\mu\left(K^{\vee}\right)$ of the dual cone $K^{\vee}$ that depends on $\Psi$, 
constructed in [PT, Cor.1]. In particular, for $\Psi$ coming from a complete flag, the constant term of $\mu^{\Psi}(K)$ gives Morelli's formula [Mo].

(6) (Inner product) For $\Psi$ coming from an inner product $Q^{*}$ on $V^{*}$, the function $\mu^{\Psi}(K)$ agrees with $\mu(\bar{K})$ constructed in $[\mathrm{BV}]$, where $\bar{K}$ is the image of $K$ in $V / V(K)$, and where $V(K)$ denotes the linear subspace parallel to the largest affine subspace contained in $K$.

There are two corollaries of Theorem 1 a local version of the Euler-Maclaurin formula for polytopes (promised in the introduction), and a meromorphic function on $\mathrm{Fl}\left(V^{*}\right) \times V^{*}$ associated to a pointed cone $K$ in $V$, where $\mathrm{Fl}\left(V^{*}\right)$ is the variety of complete flags of $V$.

To formulate these results, fix a rigid complement map $\Psi$ on $V$, a $\Psi$-generic rational convex polyhedron $P$ and a face $F$ of $P$. Consider the Taylor series expansion of $\mu^{\Psi}(\operatorname{Supp}(P, F))(\xi)$ and the corresponding differential operator (of infinite order, with constant coefficients):

$$
D^{\Psi}(P, F)=\mu^{\Psi}(\operatorname{Supp}(P, F))\left(\partial_{x}\right) .
$$

Theorem 2. Let $\Psi$ be a complement map on $V^{*}$, let $P$ be a $\Psi$-generic rational polytope in $V$, and let $h(x)$ be a polynomial function on $V$. With the above notation, we have

$$
\sum_{x \in P \cap \Lambda} h(x)=\sum_{F \in \mathcal{F}(P)} \int_{F} D^{\Psi}(P, F) \cdot h
$$

In particular, if $h(x)=1$, then

$$
\#(P)=\sum_{F \in \mathcal{F}(P)} \mu^{\Psi}(\operatorname{Supp}(P, F))(0) \operatorname{vol}(F),
$$

where $\#(P)$ (resp. $\operatorname{vol}(P))$ denotes the number of lattice points (resp. the volume) of $P$.

For concrete examples, see Section 5, Equation (15) computes the number of lattice points of a polytope as a weighted sum of the volume of its faces.

Suppose now that $\Psi$ comes from a flag $L$. Letting $L$ vary, we obtain the following.

Theorem 3. Given a pointed cone $K$ in $V$ there exists an effectively computable meromorphic function $\mu(K)$ in $\mathrm{Fl}\left(V^{*}\right) \times V^{*}$ which is regular in a Zariski open subset of $\operatorname{Fl}\left(V^{*}\right) \times\{0\}$.

For an algorithmic computation of $\mu(K)$ for cones $K$ of dimension at most 2, see Section 5 .

The next conjecture identifies the constant term of a $\Psi$-compatible interpolator with the one of $[\mathrm{PT}]$. We post it as a conjecture for now and hope to discuss it in a future publication.

Conjecture 1. Part (5) of Theorem 1 holds in all dimensions.

Question 1. Is there any relation between the rational functions $\mu(K)$ in $\mathrm{Fl}\left(V^{*}\right) \times$ $V^{*}$ and the quantum cohomology ring on $\mathrm{Fl}\left(V^{*}\right)$ ? 
3.2. IS-interpolators and reverse Euler-Maclaurin formula. The definition of the SI-interpolator leads in a natural way to the notion of an IS-interpolator, which may be a useful notion to numerical approximations of integrals by sums. The next notion requires us to restrict out attention to lattice polyhedra, i.e., polyhedra whose vertices are points of the lattice $\Lambda$. Below, a lattice cone $K$ in $V$ is a rational cone such that $U \cap \Lambda \neq \emptyset$, where $U$ is the largest affine subspace contained in $K$.

Definition 3.1. An IS-interpolator is a map $\lambda$ from the set of all lattice cones in $V$ to $\mathcal{M}\left(V^{*}\right)$ such that for any lattice polyhedron $P$ in $V$ we have

$$
I(P)=\sum_{F \in \mathcal{F}(P)} \lambda(\operatorname{Supp}(P, F)) S(F),
$$

where the sum is over all faces $F$ of $P$. An IS-interpolator is regular if $\lambda$ takes values in $\mathcal{M}^{r}\left(V^{*}\right)$.

Hereditary and $\Psi$-compatible IS-interpolators are defined in an analogous way to Definitions 2.3 and 2.10 .

The statement and proof of Theorem 1 hold with minor modifications for ISinterpolators.

Theorem 4. (a) If $V$ is a rational vector space and $\Psi$ is a rigid complement map on $V^{*}$, then there is a unique $\Psi$-compatible IS-interpolator $\lambda^{\Psi}$.

(b) In addition, $\lambda^{\Psi}$ satisfies properties (2)-(5) of Theorem 1 and the following version of additivity:

(1) (Additivity) If $K$ is a cone that does not contain a linear subspace and is subdivided into a finite union of cones $K_{i}$, then

$$
\lambda^{\Psi}(K)=\sum_{i: \operatorname{dim}\left(K_{i}\right)=\operatorname{dim}(K)} \lambda^{\Psi}\left(K_{i}\right) .
$$

One can directly obtain a formula for $\lambda^{\Psi}$ in terms of the interpolator $\mu^{\Psi}$ of Theorem 1 as follows, by observing that $\mu^{\Psi}(\operatorname{Supp}(K, K))=1$.

Theorem 5. If $K$ is a positive (resp. zero) dimensional lattice cone, then

$$
\sum_{F \in \mathcal{F}(K)} \lambda^{\Psi}(F) \mu^{\Psi}(\operatorname{Supp}(K, F))=0, \quad\left(\text { resp. } \quad \lambda^{\Psi}(K)=1\right) .
$$

The interpolator $\lambda^{\Psi}$ gives a reverse Euler-Maclaurin summation formula. Fix a rigid complement map $\Psi$ on $V$, a $\Psi$-generic lattice polyhedron $P$ and a face $F$ of $P$. Consider the Taylor series expansion of $\lambda^{\Psi}(\operatorname{Supp}(P, F))(\xi)$ and the corresponding differential operator (of infinite order, with constant coefficients):

$$
\Delta^{\Psi}(P, F)=\lambda^{\Psi}(\operatorname{Supp}(P, F))\left(\partial_{x}\right) .
$$

Theorem 6. With the above assumptions, for every lattice polytope $P$ and every polynomial function $h(x)$ on $V$ we have

$$
\int_{P} h(x)=\sum_{F \in \mathcal{F}(P)} \sum_{x \in F \cap \Lambda}\left(\Delta^{\Psi}(P, F) \cdot h\right)(x) .
$$

In particular, if $h(x)=1$, then

$$
\operatorname{vol}(P)=\sum_{F \in \mathcal{F}(P)} \lambda^{\Psi}(\operatorname{Supp}(P, F))(0) \#(F),
$$

where $\#(F)$ denotes the number of lattice points of $F$. 
Equation (20) computes the volume of a lattice polytope as a weighted sum of the number of lattice points of its faces. A formula of this type first appeared in Morelli [Mo, Eqn.(5)].

3.3. $\mathrm{IS}^{0}$-interpolators and Morelli's work. When $\Psi$ comes from a complete flag, equations (15) and (20) are similar with some results [Mo, Eqn.(5),Eqn.(6)] of Morelli. To explain this, let us introduce the variant $S^{0}$ of the exponential sum function defined by

$$
S^{0}(P)=S\left(P^{0}\right)
$$

where $P^{0}$ denotes the (relative) interior of a polyhedron $P$. The set $P^{0}$ is not a polyhedron itself; however, it is a virtual sum of polyhedra. Then, we can talk about IS $^{0}$-interpolators $\nu$ on lattice cones. Theorems 4 and 5 have the following analogue.

Theorem 7. (a) If $V$ is a rational vector space and $\Psi$ is a rigid complement map on $V^{*}$, then there is a unique $\Psi$-compatible $\mathrm{IS}^{0}$-interpolator $\nu^{\Psi}$. In addition,

(b) (Additivity) If $K$ is a cone that does not contain a linear subspace and is subdivided into a finite union of cones $K_{i}$, then $\nu^{\Psi}(K)=\sum_{i: \operatorname{dim}\left(K_{i}\right)=\operatorname{dim}(K)} \nu^{\Psi}\left(K_{i}\right)$.

(c) $\nu^{\Psi}$ satisfies properties (2)-(5) of Theorem 1.

(d) If $K$ is a lattice cone, then

$$
\sum_{F \in \mathcal{F}(K)} \nu^{\Psi}(F) \mu^{\Psi}(\operatorname{Supp}(K, F))=1 .
$$

Fix a lattice polytope $P$ and a face $F$ of $P$. If $\Psi$ comes from a complete flag $L$ in $V^{*}$ as in Lemma 2.6. and we vary the flag, we obtain two rational functions $\nu(\operatorname{Supp}(P, F))$ and $\mu(\operatorname{Supp}(P, F))$ on $\operatorname{Fl}\left(V^{*}\right) \times V^{*}$ which are regular at a Zariski open subset of $\mathrm{Fl}\left(V^{*}\right) \times 0$. Thus, we can consider the constant terms $\nu^{\Psi}(\operatorname{Supp}(P, F))(0)$ and $\mu^{\Psi}(\operatorname{Supp}(P, F))(0)$ which are rational functions on $\operatorname{Fl}\left(V^{*}\right)$.

On the other hand, Morelli constructs a pair of rational functions $\nu_{k}\left(\vartheta_{F} P\right)$ and $\mu_{k}\left(\vartheta_{F} P\right)$ (where $k$ is the dimension of $\operatorname{lin}(\operatorname{Supp}(P, F))$ ) that appear respectively in Equations (5) and (6) of $\mathrm{Mo}$ (unfortunately, Morelli denotes the two functions with the same notation, although he clearly recognizes that they are distinct functions). Morelli's construction uses $K$-theory, localization and the Bott residue theorem. (5).

The following proposition is a re-expression of the flag case of Theorem 1 part

Proposition 3.2. With the above notation, for polytopes $P$ of dimension at most 2, we have

$$
\nu(\operatorname{Supp}(P, F))(0)=\nu_{k}\left(\vartheta_{F} P\right), \quad \mu(\operatorname{Supp}(P, F))(0)=\mu_{k}\left(\vartheta_{F} P\right) .
$$

In [Mo, p.191] and also in [Mo, Thm.5], Morelli observes that

$$
\mu(K)(0)=\nu\left(K^{\vee}\right)(0)
$$

for lattice cones $K$ of dimension 4 or less, and that equation (24) fails in dimensions more than 4. This mysterious and seemingly deep coincidence is easy to explain, from our point of view, for cones of dimension at most 2. The rational functions $\nu(K)$ and $\mu\left(K^{\vee}\right)$ are distinct; in fact, they are functions on distinct spaces $\left(\mathrm{Fl}\left(V^{*}\right) \times\right.$ $V^{*}$ and $\operatorname{Fl}(V) \times V$, respectively). Their constant terms coincidentally agree in small dimensions. For a discussion, see Section 6.2. 


\section{Proofs}

4.1. Proof of Theorem 1, Fix a rigid complement map $\Psi$ on a rational vector space $V$ with lattice $\Lambda$ and consider a $\Psi$-compatible interpolator $\mu^{\Psi}$ and a $\Psi$-generic rational polytope $P$. We will show by induction on the dimension of $P$ the existence and uniqueness of $\mu^{\Psi}(P)$. All cones in this section will be assumed to be $\Psi$-generic. By Lemma 2.4 it suffices to assume that $P=K$ is a cone in $V$. To avoid confusion, we denote the map $\mu: \mathcal{C}^{\Psi}(V) \longrightarrow \mathcal{M}\left(V^{*}\right)$ by $\mu_{V}$ and for every $\Psi$-generic quotient $W$ of $V$, we denote the map $\bar{\mu}: \mathcal{C}^{\bar{\Psi}}(W) \longrightarrow \mathcal{M}\left(W^{*}\right)$ by $\mu_{W}$.

If $K$ is pointed with vertex $v$, then we can single out the contribution from the 0 -dimensional face of $K$ in equation (10), and together with the hereditary property of equation (11), it follows that

$$
\begin{aligned}
\mu_{V}^{\Psi}(K)(\xi)= & e^{-\langle\xi, v\rangle}\left(\sum_{x \in K \cap \Lambda} e^{\langle\xi, x\rangle}\right. \\
& \left.-\sum_{F, \operatorname{dim}(F)>0} \mu_{V / \operatorname{lin}(F)}^{\bar{\Psi}}(\overline{\operatorname{Supp}(K, F)})(\pi(\xi)) \int_{F} e^{\langle\xi, x\rangle} d m_{F}(x)\right),
\end{aligned}
$$

where $\overline{\operatorname{Supp}(K, F)}$ denotes the image of $\operatorname{Supp}(P, F)$ in the quotient space $V / \operatorname{lin}(F)$, and $\pi: V^{*} \longrightarrow(V / \operatorname{lin}(F))^{*}$ is the projection using the decomposition $V^{*}=$ $(V / \operatorname{lin}(F))^{*} \oplus \Psi\left((V / \operatorname{lin}(F))^{*}\right)$ given by the rigid complement map $\Psi$.

On the other hand, if $K$ is a non-pointed cone in $V$, let $V(K)$ denote the linear subspace of $V$ parallel to the largest affine subspace contained in $K$, and consider the image $\bar{K}$ of $K$ in $V / V(K)$, which is a pointed cone of dimension strictly less than the dimension of $K$. Equations (11) and (12) imply that

$$
\mu_{V}^{\Psi}(K)(\xi)=\mu_{V / V(K)}^{\bar{\Psi}}(\bar{K})(\bar{\xi}),
$$

where $\xi \in V^{*}, \pi: V^{*} \longrightarrow(V / V(K))^{*}$ is the projection using the decomposition $V^{*}=(V / V(K))^{*} \oplus \Psi\left((V / V(K))^{*}\right)$ given by the rigid complement map $\Psi$ and $\bar{\xi} \in(V / V(K))^{*}$ is the image of $\xi$ under the above projection.

Equations (25) and (26) uniquely define $\mu_{V}^{\Psi}$ from $\mu_{W}^{\Psi}$ for $\operatorname{dim}(W)<\operatorname{dim}(V)$. On the other hand, when $V$ is a 0 -dimensional space, we have $\mu_{V}^{\Psi}(\{0\})=1$. This uniquely determines $\mu_{V}^{\Psi}$. An explicit computation of $\mu^{\Psi}(K)$ for cones $K$ of dimensions 0,1 and 2 is given in Section 5 .

Additivity of $\mu^{\Psi}$ follows from the above inductive definition of $\mu^{\Psi}$ and the additivity (property (A2)) of the exponential sum and exponential integrals. This is presented in detail in the proof of [BV, Prop.15].

Remark 4.1. In fact, Berline-Vergne in $\mathrm{BV}$ ] work with the transverse cone $\mathrm{T}(K, F)$ of a face $F$ of $K$ defined to be the image of $\operatorname{Supp}(K, F)$ under the projection map $V \longrightarrow V / \operatorname{lin}(F)$. The transverse cone $\mathrm{T}(K, F)$ is always a pointed cone. In fact this gives a 1-1 correspondence

$$
\{\text { cones in } V\} \leftrightarrow\{\text { pointed cones in quotients of } V\} \text {. }
$$

Using this correspondence, and an inner product $Q$ on $V$, Berline-Vergne inductively construct $\mu^{Q}$ defined on the set of pointed cones on the quotients of $V$. For visual reasons, we prefer to work with the supporting cones $\operatorname{Supp}(K, F)$ rather than the transverse cones $\mathrm{T}(K, F)$. 
Going back to the proof of Theorem 1 the lattice invariance and the isometry equivariance of $\mu^{\Psi}$ follows easily by induction and the invariance of the $I$ and $S$ functions.

The regularity of the meromorphic function $\mu^{\Psi}(K)$ at zero follows by induction and a residue calculation, discussed in detail in [BV, Prop.18], using the following fact about the exponential sum and integral functions.

(A6) If $K$ is a cone in $V$ with primitive integral generators $v_{1}, \ldots, v_{k}$, then

$$
\prod_{i=1}^{k}\left\langle\xi, v_{i}\right\rangle S(K)(\xi), \quad \prod_{i=1}^{k}\left\langle\xi, v_{i}\right\rangle I(K)(\xi)
$$

are regular functions and the residues of the meromorphic functions $S(K)$ and $I(K)$ along the hyperplane $v_{1}=0$ satisfy the equations

$$
\operatorname{Res}_{v_{1}}(S(K))=-S(\bar{K}), \quad \operatorname{Res}_{v_{1}}(I(K))=-I(\bar{K}) .
$$

(A6) follows in turn by additivity and equation (8) which evaluates the $I$ and $S$ functions on simplicial cones.

We defer the proof of property (5) of Theorem 1 until the example section (Section 5). See the proofs immediately following Examples 5.6 and 5.7

Finally, property (6) of Theorem 1 follows directly from [BV, Eq. 4].

4.2. Proof of Theorem 2, Fix a polynomial function $h(x)$ on $V$ and a polytope $P$ on $V$. Consider the constant coefficient differential operator $h\left(\partial_{\xi}\right)$ which acts on the function $\xi \mapsto e^{\langle\xi, x\rangle}$ by

$$
h\left(\partial_{\xi}\right) e^{\langle\xi, x\rangle}=h(x) e^{\langle\xi, x\rangle} .
$$

The definition (7) of $S(P)$ and the above gives that

$$
h\left(\partial_{\xi}\right) S(P)=\sum_{x \in P \cap \Lambda} h(x) e^{\langle\xi, x\rangle} .
$$

Let ev denote the evaluation at $\xi=0$. It follows that

$$
\left(\mathrm{ev} \circ h\left(\partial_{\xi}\right)\right) S(P)=\sum_{x \in P \cap \Lambda} h(x) .
$$

On the other hand, Theorem 1 gives that

$$
\begin{aligned}
S(P)(\xi) & =\sum_{F \in \mathcal{F}(P)} \mu^{\Psi}(\mathrm{T}(P, F))(\xi) I(F)(\xi) \\
& =\sum_{F \in \mathcal{F}(P)} \int_{F} \mu^{\Psi}(\mathrm{T}(P, F))(\xi) e^{\langle\xi, x\rangle} d m_{P}(x) \\
& =\sum_{F \in \mathcal{F}(P)} \int_{F} \mu^{\Psi}(\mathrm{T}(P, F))\left(\partial_{x}\right) e^{\langle\xi, x\rangle} d m_{P}(x) \\
& =\sum_{F \in \mathcal{F}(P)} \int_{F} D^{\Psi}(P, F) e^{\langle\xi, x\rangle} d m_{P}(x),
\end{aligned}
$$

where the last equality follows from the definition (13) of the differential operator $D^{\Psi}(P, F)$. Applying the differential operator $h\left(\partial_{\xi}\right)$ to both sides it follows that

$$
h\left(\partial_{\xi}\right) I(P)(\xi)=\sum_{F \in \mathcal{F}(P)} \int_{F} D^{\Psi}(P, F) h(x) e^{\langle\xi, x\rangle} d m_{P}(x) .
$$


Evaluating at $\xi=0$, it follows that

$$
\left(\mathrm{ev} \circ h\left(\partial_{\xi}\right)\right) S(P)=\sum_{F \in \mathcal{F}(P)} \int_{F} D^{\Psi}(P, F) \cdot h .
$$

Equations (31) and (32) complete the proof of Theorem 2,

4.3. Proof of Theorem 4. The proof of Theorem 1 applies verbatim, with the following observation, which explains the need for lattice polyhedra.

If $K$ is a rational pointed cone with vertex $v$ for which (16) applies, then one of the terms in (16) is $F=\{v\}$. In that case, $\mathrm{T}(K,\{v\})=K$ and the corresponding term in (16) is $\nu(K) S(\{v\})$. Now, (A3) and (A5) imply that

$$
S(\{v\})(\xi)= \begin{cases}e^{\langle\xi, v\rangle} & \text { if } v \in \Lambda, \\ 0 & \text { otherwise. }\end{cases}
$$

Thus, when $K$ is not a lattice cone, then we cannot solve for $\nu(K)$. In the case of SI-interpolators, as discussed in Section 2.4, the corresponding term of (10) was $\mu(K) I(\{v\})$ and (A3) and (A5) imply that

$$
I(\{v\})(\xi)=e^{\langle\xi, v\rangle} .
$$

4.4. Proof of Theorems 5 and 7 , Theorem 5 follows from the fact that the composition of an SI-interpolator with an IS-interpolator is an SS-interpolator, and the fact that an SS-interpolator is unique. More precisely, fix an SI-interpolator $\mu$ and an IS-interpolator $\lambda$, and consider a lattice cone $K$. Then, on the one hand, we have

$$
S(K)=\sum_{F^{\prime} \in \mathcal{F}(K)} \mu\left(\operatorname{Supp}\left(K, F^{\prime}\right)\right) I\left(F^{\prime}\right) .
$$

On the other hand, for every face $F^{\prime}$ of $K$, we have

$$
I\left(F^{\prime}\right)=\sum_{F \in \mathcal{F}\left(F^{\prime}\right)} \lambda\left(\operatorname{Supp}\left(F^{\prime}, F\right)\right) S(F) .
$$

Substituting the second equation into the first, it follows that

$$
S(K)=\sum_{F, F^{\prime}: F \subset F^{\prime}} \mu\left(\operatorname{Supp}\left(K, F^{\prime}\right)\right) \lambda\left(\operatorname{Supp}\left(F^{\prime}, F\right)\right) S(F) .
$$

This motivates the following definition. Consider the function

$$
\operatorname{Supp}(P, F) \mapsto(\lambda \circ \mu)(\operatorname{Supp}(P, F)):=\sum_{F^{\prime}: F \subset F^{\prime}} \mu\left(\operatorname{Supp}\left(K, F^{\prime}\right)\right) \lambda\left(\operatorname{Supp}\left(F^{\prime}, F\right)\right) .
$$

It is easy to see that $\lambda \circ \mu$ is an SS-interpolator. Moreover, if $\mu$ and $\lambda$ are $\Psi$ compatible, so is their composition. On the other hand, there is a unique $\Psi$ compatible SS-interpolator. Thus $(\lambda \circ \mu)(\operatorname{Supp}(P, F))=0$ (resp. 1$)$ for $F \neq P$ (resp. $F=P$ ). This concludes the proof of Theorem 5 .

Theorem 7 follows by an analogous computation, using the fact that the map $(\nu \circ \mu)$ is a $\Psi$-compatible $\mathrm{SS}^{0}$-intepolator, the uniqueness of such interpolators, and the fact that the map that sends every cone to 1 is a $\Psi$-compatible $\mathrm{SS}^{0}$-intepolator. 


\section{Computations And EXAmples}

5.1. Computation of $\mu^{\Psi}$ for low-dimensional cones. As was mentioned earlier, the meromorphic functions $\mu^{\Psi}(K)$ are effectively computable given a complement map $\Psi$. In this section we illustrate this by explicitly computing $\mu^{\Psi}(K)$ for cones $K$ of dimension at most 2 .

Proposition 5.1. Suppose that $K=\{0\}$ in the vector space $V=\{0\}$. Then $\mu(K)$ is the constant function 1 , independent of $\Psi$.

Proof. $S(K)=I(K)=1$ forces $\mu(K)=1$.

If $\left\{v_{1}, \ldots, v_{k}\right\}$ is a collection of vectors in $V$, let $\left(v_{1}, \ldots, v_{k}\right)=\mathbb{Q}^{+} v_{1}+\cdots+\mathbb{Q}^{+} v_{k}$ denote the cone spanned by those vectors, where $\mathbb{Q}^{+}$is the set of non-negative rational numbers.

Proposition 5.2. Now suppose that $V$ is 1 -dimensional and $K=\operatorname{Cone}(v)$ is a ray in $V$ generated by a primitive vector $v \in \Lambda$. Then, independent of $\Psi$, we have

$$
\mu(K)(\xi)=B(\langle\xi, v\rangle)
$$

where

$$
B(z)=\frac{1}{1-e^{z}}+\frac{1}{z}=\frac{1}{2}-\frac{z}{12}+\frac{z^{3}}{720}-\frac{z^{5}}{30240}+\ldots
$$

is the generating series of the Bernoulli numbers.

Proof. Using the property of interpolators

$$
S(K)=\mu(\operatorname{Supp}(K, K)) I(K)+\mu(\operatorname{Supp}(K, 0)) I(0),
$$

$\mu(\operatorname{Supp}(K, K))=1$ by the previous proposition and compatibility under quotients. In addition, we have $S(K)(\xi)=1 /\left(1-e^{\xi}\right)$ and $I(K)(\xi)=-1 / \xi$. The result follows.

For the following proposition, recall that if $K$ is a subset of $V$, then we define the dual $K^{\vee}$ by

$$
K^{\vee}=\left\{w \in V^{*} \mid\langle w, v\rangle \geq 0 \text {, for all } v \in K\right\} .
$$

Also, if $\Lambda$ is a lattice in $V$, then $\Lambda^{*}=\left\{w \in V^{*} \mid\langle w, v\rangle \in \mathbb{Z}\right.$, for all $\left.v \in \Lambda\right\}$ is the dual lattice in $V^{*}$. Below, we will denote by $\mu^{L}$ (resp. $\mu^{Q}$ ) the $\Psi$-compatible SIinterpolator, where $\Psi$ comes from a complete flag $L$ on $V$ (resp. an inner product $Q$ on $V$ ), as in Lemma 2.6.

Proposition 5.3. Suppose that $V$ is 2-dimensional and that $K \subset V$ is a half-plane with boundary a line $U$ through the origin. Let $\rho \in \Lambda^{*}$ be the primitive generator of the ray $K^{\vee}$. Suppose that $\Psi$ is defined by a complete flag $L$ in $V^{*}$, and let $c$ be a generator of $L_{1}^{*}$, where $L_{1}$ is the 1-dimensional subspace of the flag $L$ (the only non-trivial subspace in the flag). Then

$$
\mu^{L}(K)(\xi)=B\left(\frac{\langle\xi, c\rangle}{\langle\rho, c\rangle}\right) .
$$

Now suppose that $\Psi$ is defined by an inner product $Q$ on $V$. Then

$$
\mu^{Q}(K)(\xi)=B\left(\frac{Q(\xi, \rho)}{Q(\rho, \rho)}\right)
$$



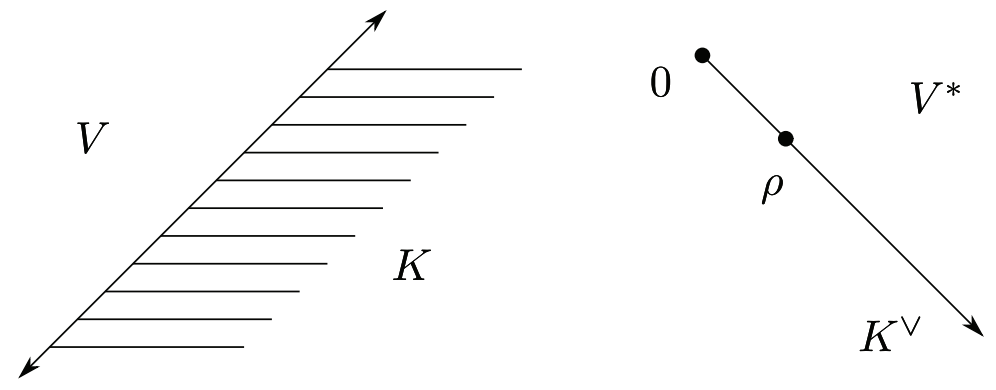

FiguRE 4

Proof. We use the $\Psi$-compatibility together with our 1-dimensional formula above. To do so, we must compute the projection from $\pi: V^{*} \rightarrow W^{*}$, where $W=V / U$. This projection is computed using the decomposition $V^{*}=W^{*} \oplus \Psi^{*}\left(W^{*}\right)$ given by the complement map $\Psi$. Suppose that the 1-dimensional subspace $\Psi\left(W^{*}\right) \subset V^{*}$ is generated by $d$. Then for $\xi \in V^{*}$, find scalars $\omega_{1}, \omega_{2}$ such that

$$
\xi=\omega_{1} \rho+\omega_{2} d .
$$

Then from $\Psi$-compatibility and the 1-dimensional formula, we obtain

$$
\mu(K)(\xi)=B\left(\omega_{1}\right) .
$$

For $\Psi$ coming from a complete flag, we pair both sides of equation (35) with the generator $c$. Since $\langle d, c\rangle=0$, we find

$$
\omega_{1}=\frac{\langle\xi, c\rangle}{\langle\rho, c\rangle},
$$

and the formula for $\mu^{L}(K)$ follows. For $\Psi$ coming from the inner product $Q$, we note that $Q(d, \rho)=0$. Thus equation (35) yields

$$
\omega_{1}=\frac{Q(\xi, \rho)}{Q(\rho, \rho)},
$$

which completes the proof.

Using the defining property of interpolators, we immediately get the following proposition, an explicit expression for $\mu$ of a non-singular 2-dimensional cone.

Proposition 5.4. Suppose $V$ is 2-dimensional and $K=$ Cone $\left(v_{1}, v_{2}\right)$, where $v_{1}, v_{2}$ form a basis of $\Lambda \subset V$. Let $F_{1}=\operatorname{Cone}\left(v_{1}\right)$ and $F_{2}=\operatorname{Cone}\left(v_{2}\right)$, and let $\rho_{1}, \rho_{2} \in \Lambda^{*}$ denote the primitive normals to $F_{1}$ and $F_{2}$. Then for $\Psi$ coming from a complete flag $L$ in $V^{*}$, we have

$$
\begin{aligned}
\mu^{L}(K)= & \frac{1}{\left(1-e^{\left\langle\xi, v_{1}\right\rangle}\right)\left(1-e^{\left\langle\xi, v_{2}\right\rangle}\right)}-\frac{1}{\left\langle\xi, v_{1}\right\rangle\left\langle\xi, v_{2}\right\rangle}+\frac{1}{\left\langle\xi, v_{1}\right\rangle} B\left(\frac{\langle\xi, c\rangle}{\left\langle\rho_{1}, c\right\rangle}\right) \\
& +\frac{1}{\left\langle\xi, v_{2}\right\rangle} B\left(\frac{\langle\xi, c\rangle}{\left\langle\rho_{2}, c\right\rangle}\right) .
\end{aligned}
$$


For $\Psi$ coming from an inner product $Q$ on $V^{*}$, we have

$$
\begin{aligned}
\mu^{Q}(K)= & \frac{1}{\left(1-e^{\left\langle\xi, v_{1}\right\rangle}\right)\left(1-e^{\left\langle\xi, v_{2}\right\rangle}\right)}-\frac{1}{\left\langle\xi, v_{1}\right\rangle\left\langle\xi, v_{2}\right\rangle}+\frac{1}{\left\langle\xi, v_{1}\right\rangle} B\left(\frac{Q\left(\xi, \rho_{1}\right)}{Q\left(\rho_{1}, \rho_{1}\right)}\right) \\
& +\frac{1}{\left\langle\xi, v_{2}\right\rangle} B\left(\frac{Q\left(\xi, \rho_{2}\right)}{Q\left(\rho_{2}, \rho_{2}\right)}\right) .
\end{aligned}
$$

Remark 5.5. Replacing $1 /\left(1-e^{x}\right)$ with $B(x)-1 / x$, it follows that equations (36) and (37) can be written in the form:

$$
\begin{aligned}
\mu^{L}(K)= & B\left(\left\langle\xi, v_{1}\right\rangle\right) B\left(\left\langle\xi, v_{2}\right\rangle\right)+\frac{1}{\left\langle\xi, v_{1}\right\rangle}\left(B\left(\frac{\langle\xi, c\rangle}{\left\langle\rho_{1}, c\right\rangle}\right)-B\left(\left\langle\xi, v_{2}\right\rangle\right)\right) \\
& +\frac{1}{\left\langle\xi, v_{2}\right\rangle}\left(B\left(\frac{\langle\xi, c\rangle}{\left\langle\rho_{2}, c\right\rangle}\right)-B\left(\left\langle\xi, v_{1}\right\rangle\right)\right) \\
\mu^{Q}(K)= & B\left(\left\langle\xi, v_{1}\right\rangle\right) B\left(\left\langle\xi, v_{2}\right\rangle\right)+\frac{1}{\left\langle\xi, v_{1}\right\rangle}\left(B\left(\frac{Q\left(\xi, \rho_{1}\right)}{Q\left(\rho_{1}, \rho_{1}\right)}\right)-B\left(\left\langle\xi, v_{2}\right\rangle\right)\right) \\
& +\frac{1}{\left\langle\xi, v_{2}\right\rangle}\left(B\left(\frac{Q\left(\xi, \rho_{2}\right)}{Q\left(\rho_{2}, \rho_{2}\right)}\right)-B\left(\left\langle\xi, v_{1}\right\rangle\right)\right) .
\end{aligned}
$$

5.2. Examples. In this section we explicitly compute some low-dimensional examples and match them with the ones given by Morelli, Pommersheim-Thomas and Berline-Vergne.

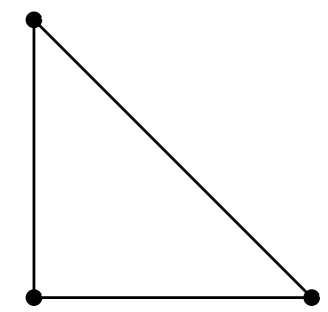

Figure 5

Example 5.6. Consider the triangle $P$ shown in Figure 5 with vertices $v_{0}=$ $(0,0), v_{1}=(1,0), v_{2}=(0,1)$ in the lattice $\Lambda=\mathbb{Z}^{2} \subset V=\mathbb{Q}^{2}$, where $V^{*}$ is also identified with $\mathbb{Z}^{2}$. Let $\Psi$ be the complement map coming from the complete flag $L=\left(L_{0}, L_{1}, L_{2}\right)$ in $V^{*}$ such that $L_{1}=\operatorname{Span}\left\{\left(d_{1}, d_{2}\right)\right\}$. To compute $\mu^{L}$ of the supporting cones, we use lattice invariance to translate them to the origin. In other words, consider the tangent cones $K_{i}=\operatorname{Tan}\left(P, v_{i}\right)$ for $i=0,1,2$, to $P$ at $v_{i}$. Specifically, we have

$$
\begin{aligned}
& K_{0}=\operatorname{Cone}((1,0),(0,1)), \\
& K_{1}=\operatorname{Cone}((-1,1),(-1,0)), \\
& K_{2}=\operatorname{Cone}((0,-1),(1,-1)) .
\end{aligned}
$$


Then for $\xi=\left(\xi_{1}, \xi_{2}\right) \in V^{*}$, we have

$$
\begin{aligned}
\mu^{L}\left(K_{0}\right)(\xi)= & \frac{1}{\left(1-e^{\xi_{1}}\right)\left(1-e^{\xi_{2}}\right)}-\frac{1}{\xi_{1} \xi_{2}}+\frac{1}{\xi_{2}} B\left(\xi_{1}-\frac{d_{1}}{d_{2}} \xi_{2}\right)+\frac{1}{\xi_{1}} B\left(\xi_{2}-\frac{d_{2}}{d_{1}} \xi_{1}\right) \\
\mu^{L}\left(K_{1}\right)(\xi)= & \frac{1}{\left(1-e^{\xi_{2}-\xi_{1}}\right)\left(1-e^{-\xi_{1}}\right)}+\frac{1}{\xi_{1}\left(\xi_{2}-\xi_{1}\right)}-\frac{1}{\xi_{1}} B\left(\xi_{2}-\frac{d_{2}}{d_{1}} \xi_{1}\right) \\
& +\frac{1}{\xi_{2}-\xi_{1}} B\left(\frac{d_{2} \xi_{1}-d_{1} \xi_{2}}{d_{1}-d_{2}}\right) \\
\mu^{L}\left(K_{2}\right)(\xi)= & \frac{1}{\left(1-e^{\xi_{1}-\xi_{2}}\right)\left(1-e^{-\xi_{2}}\right)}+\frac{1}{\xi_{2}\left(\xi_{1}-\xi_{2}\right)}-\frac{1}{\xi_{2}} B\left(\xi_{1}-\frac{d_{1}}{d_{2}} \xi_{2}\right) \\
& +\frac{1}{\xi_{1}-\xi_{2}} B\left(\frac{d_{2} \xi_{1}-d_{1} \xi_{2}}{d_{1}-d_{2}}\right) .
\end{aligned}
$$

These functions are analytic at $\xi=(0,0)$. For example, $\mu^{L}\left(K_{0}\right)(\xi)$ has the Taylor expansion

$$
\begin{aligned}
\mu^{L}\left(K_{0}\right)(\xi)=\left(\frac{d_{1}^{2}+d_{2}^{2}+3 d_{1} d_{2}}{12 d_{1} d_{2}}\right) & +\left(\frac{-1}{24}\right) \xi_{1}+\left(\frac{-1}{24}\right) \xi_{2} \\
& +\left(\frac{3 d_{1}^{4}+3 d_{2}^{4}+5 d_{1}^{2} d_{2}^{2}}{720 d_{1}^{2} d_{2}^{2}}\right) \xi_{1} \xi_{2} \\
& +\left(\frac{-3 d_{1}^{4}-d_{2}^{4}}{720 d_{1}^{3} d_{2}}\right) \xi_{1}^{2}+\left(\frac{-3 d_{2}^{4}-d_{1}^{4}}{720 d_{2}^{3} d_{1}}\right) \xi_{2}^{2}+\cdots
\end{aligned}
$$

Denoting the constant term of each $\mu^{L}\left(K_{i}\right)$ by $\mu_{0}^{L}\left(K_{i}\right)$, we find

$$
\begin{aligned}
& \mu_{0}^{L}\left(K_{0}\right)=\frac{d_{1}^{2}+d_{2}^{2}+3 d_{1} d_{2}}{12 d_{1} d_{2}}, \\
& \mu_{0}^{L}\left(K_{1}\right)=\frac{5 d_{1}^{2}-5 d_{1} d_{2}+d_{2}^{2}}{12 d_{1}\left(d_{1}-d_{2}\right)}, \\
& \mu_{0}^{L}\left(K_{2}\right)=\frac{5 d_{2}^{2}-5 d_{1} d_{2}+d_{1}^{2}}{12 d_{2}\left(d_{2}-d_{1}\right)}
\end{aligned}
$$

in exact agreement with Morelli's example [Mo pp.198-199]. These rational functions can also be computed by multiplying the Todd polynomials using $[\mathrm{PT}$, Theorem 3]. In particular, these three rational functions sum to 1 in agreement with equation (15).

In addition, one checks by direct computation that

$$
\sum_{F} \mu^{L}(\operatorname{Supp}(P, F)) I(F)=S(P)=1+e^{\xi_{1}}+e^{\xi_{2}}
$$

in agreement with the definition of an interpolator.

Indeed, this example provides a general proof of the coincidence of our construction and the flag case of $[\mathrm{PT}]$ for cones of dimension at most 2, and hence the coincidence with Morelli's formulas.

Proof of 1, part (5) for flags. In dimension 1, the [PT] values are always equal to $\frac{1}{2}$, independent of the complement map. Using Proposition 5.2 we see that this coincides with the constant term of $\mu$. For the 2-dimensional case, we first use the additivity of $[\mathrm{PT}]$ and of our construction (Theorem 1, property (1)) to reduce to non-singular 2-dimensional cones. By functoriality, we may assume that $K$ is the 
cone $K_{0}$ from Example 5.6 generated by the standard basis of $\mathbb{Z}^{2}$. In this case, we note that the values of $\mu_{0}^{L}\left(K_{0}\right)$ given in Example 5.6 match the values in Mo, pp.198-199]. By [PT, Corollary 2], these values also match the values in [PT]. This completes the proof.

Example 5.7. We consider the same triangle $P$ from Example 5.6, but now with a complement map $\Psi$ coming from an inner product $Q$ on $V^{*}$, given by the matrix

$$
\left(\begin{array}{ll}
a & b \\
b & c
\end{array}\right)
$$

We can use Proposition 5.4 to compute $\mu^{Q}\left(K_{i}\right)$ for the supporting cones $K_{i}$ of $P$. We find

$$
\begin{aligned}
\mu^{Q}\left(K_{0}\right)(\xi)= & \frac{1}{\left(1-e^{\xi_{1}}\right)\left(1-e^{\xi_{2}}\right)}-\frac{1}{\xi_{1} \xi_{2}}+\frac{1}{\xi_{2}} B\left(\xi_{1}+\frac{b}{a} \xi_{2}\right)+\frac{1}{\xi_{1}} B\left(\xi_{2}+\frac{b}{c} \xi_{1}\right) \\
\mu^{Q}\left(K_{1}\right)(\xi)= & \frac{1}{\left(1-e^{\xi_{2}-\xi_{1}}\right)\left(1-e^{-\xi_{1}}\right)}+\frac{1}{\xi_{1}\left(\xi_{2}-\xi_{1}\right)}-\frac{1}{\xi_{1}} B\left(\xi_{2}+\frac{b}{c} \xi_{1}\right) \\
& +\frac{1}{\xi_{2}-\xi_{1}} B\left(\frac{-a \xi_{1}-b \xi_{1}-b \xi_{2}-c \xi_{2}}{a+2 b+c}\right) \\
\mu^{Q}\left(K_{2}\right)(\xi)= & \frac{1}{\left(1-e^{\xi_{1}-\xi_{2}}\right)\left(1-e^{-\xi_{2}}\right)}+\frac{1}{\xi_{2}\left(\xi_{1}-\xi_{2}\right)}-\frac{1}{\xi_{2}} B\left(\xi_{1}+\frac{b}{a} \xi_{2}\right) \\
& +\frac{1}{\xi_{1}-\xi_{2}} B\left(\frac{-a \xi_{1}-b \xi_{1}-b \xi_{2}-c \xi_{2}}{a+2 b+c}\right)
\end{aligned}
$$

This time, we find that the constant terms are given by

$$
\begin{aligned}
& \mu_{0}^{Q}\left(K_{0}\right)=\frac{3 a c-a b-b c}{12 a c}, \\
& \mu_{0}^{Q}\left(K_{1}\right)=\frac{a b+4 a c+10 b c+2 b^{2}+5 c^{2}}{12\left(a c+2 b c+c^{2}\right)}, \\
& \mu_{0}^{Q}\left(K_{2}\right)=\frac{5 a^{2}+2 b^{2}+10 a b+4 a c+b c}{12\left(a^{2}+2 a b+a c\right)} .
\end{aligned}
$$

These agree with the values of $\mu$ constructed in [PT, Cor.1] for $\Psi$ coming from an inner product. In particular, the three rational functions above sum to 1 . For the standard inner product on $V^{*}=\mathbb{Z}^{2}$ (corresponding to $a=c=1, b=0$ ), we find

$$
\mu_{0}^{Q}\left(K_{0}\right)=\frac{1}{4}, \quad \mu_{0}^{Q}\left(K_{1}\right)=\frac{3}{8}, \quad \mu_{0}^{Q}\left(K_{2}\right)=\frac{3}{8} .
$$

For completeness and comparison, we detail how these numbers arise, in a seemingly very different way, out of the construction of Pommersheim-Thomas. We begin, according to $[\mathrm{PT}$, Cor.1(iv)] by considering the outer normal fan of $P$. The rays of this fan are $\rho_{0}=(1,1), \rho_{1}=(-1,0)$, and $\rho_{2}=(0,-1)$. We must then multiply the second-degree Todd polynomial $T=\sum_{i<j} D_{i} D_{j}+\frac{1}{12} \sum_{i} D_{i}^{2}$ in the ring given in [PT, Prop.2]. In this ring, one finds $D_{0}^{2}=\frac{1}{2} D_{0} D_{1}+\frac{1}{2} D_{0} D_{2}, D_{1}^{2}=D_{0} D_{1}$, and $D_{2}^{2}=D_{0} D_{2}$. Hence

$$
T=\frac{1}{4} D_{1} D_{2}+\frac{3}{8} D_{0} D_{2}+\frac{3}{8} D_{0} D_{1}
$$

from which one reads off the values of $\mu_{0}\left(K_{0}\right)=\frac{1}{4}, \mu_{0}\left(K_{1}\right)=\frac{3}{8}$, and $\mu_{0}\left(K_{2}\right)=\frac{3}{8}$. In fact, more generally, let $K=\operatorname{cone}\left(v_{1}, v_{2}\right)$ be any non-singular cone in $V$ with 
vertex at 0 , and let $Q$ be an inner product on $V^{*}$. Then an easy computation similar to the above can be used to compute the value of $\mu_{0}$ of $[\mathrm{PT}]$ for this cone $K$. Indeed, if $K^{*}=\operatorname{cone}\left(\rho_{1}, \rho_{2}\right)$ is the dual cone, then using the presentation of PT, Proposition 2], one finds that the coefficient of $D_{1} D_{2}$ in $T$ is given by (38)

$\frac{1}{4}-\frac{Q\left(\rho_{1}, \rho_{2}\right)}{12}\left(\frac{1}{Q\left(\rho_{1}, \rho_{1}\right)}+\frac{1}{Q\left(\rho_{2}, \rho_{2}\right)}\right)=\frac{1}{4}+\frac{Q^{*}\left(v_{1}, v_{2}\right)}{12}\left(\frac{1}{Q^{*}\left(v_{1}, v_{1}\right)}+\frac{1}{Q^{*}\left(v_{2}, v_{2}\right)}\right)$, where $Q^{*}$ denotes the dual inner product on $V$.

Indeed, this calculation proves the inner product case of Theorem 11 part (5), as we now show.

Proof of Theorem [1, part (5) for inner products. As in the flag case, the [PT] values are always equal to $\frac{1}{2}$, independent of the complement map, matching the constant term of the functions in Proposition 5.2. For the 2-dimensional case, we again use additivity and functoriality to reduce to the case in which $K$ is the cone $K_{0}$ from Example 5.6. We then note that the formula of equation 38 for the $\left.\mathrm{PT}\right]$ construction matches the values of $\mu_{0}^{Q}\left(K_{0}\right)$ given in Example 5.7. This completes the proof.

A comparison of the functions $\mu_{0}^{Q}\left(K_{i}\right)$ of Example 5.7 with the functions $\mu_{0}^{L}\left(K_{i}\right)$ of Example 5.6 reveals that they are indeed different, confirming the fact that interpolators that come from flags are different from those that come from an inner product.

Let us repeat the previous example with a different triangle.

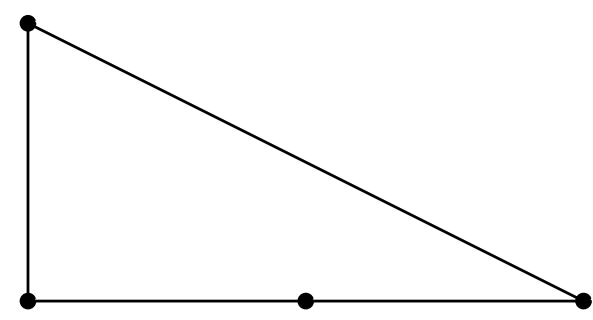

FiguRE 6

Example 5.8. Consider the triangle $P$ shown in Figure 6 with vertices $v_{0}=$ $(0,0), v_{1}=(2,0), v_{2}=(0,1)$. As above, let $\Psi$ be the complement map coming from the complete flag $L=\left(L_{0}, L_{1}, L_{2}\right)$ in $V^{*}$ such that $L_{1}^{*}=\operatorname{Span}\left\{\left(d_{1}, d_{2}\right)\right\}$. Denoting, as before, the tangent cones at the vertex $v_{i}$ by $K_{i}$, we see that $K_{0}=$ Cone $((1,0),(0,1))$, and $K_{1}=\operatorname{Cone}((-1,0),(-2,1))$ are non-singular cones, whose $\mu$ values may be computed from Proposition 5.4. On the other hand, the cone $K_{2}=\operatorname{Cone}((0,-1),(2,-1))$ is singular. We can compute $\mu^{L}\left(K_{2}\right)$ by subdividing $K_{2}$ into two cones:

$$
K_{2}=C_{1} \cup C_{2}, \quad C_{1}=\operatorname{Cone}((0,-1),(1,-1)), \quad C_{2}=\operatorname{Cone}((1,-1),(1,-2))
$$

using the ray $\rho=\operatorname{Cone}((1,-1))$. The additivity of $\mu$ implies that

$$
\mu^{L}\left(K_{2}\right)=\mu^{L}\left(C_{1}\right)+\mu^{L}\left(C_{2}\right)-\mu^{L}(\rho) .
$$


The terms on the right-hand side can be computed using Propositions 5.4 and 5.2. Alternatively, one can compute $\mu^{L}\left(K_{2}\right)$ directly from the defining formula for interpolators. Letting $F_{1}=\operatorname{Cone}((0,-1))$ and $F_{2}=\operatorname{Cone}((2,-1))$ denote the 1-dimensional faces of $K_{2}$, we get

$$
\begin{aligned}
\mu^{L}\left(K_{2}\right)= & S\left(K_{2}\right)-I\left(K_{2}\right)-\mu^{L}\left(\operatorname{Supp}\left(K_{2}, F_{1}\right)\right) I\left(F_{1}\right)-\mu^{L}\left(\operatorname{Supp}\left(K_{2}, F_{2}\right)\right) I\left(F_{2}\right) \\
= & \frac{1+e^{\xi_{1}-\xi_{2}}}{\left(1-e^{-\xi_{2}}\right)\left(1-e^{2 \xi_{1}-\xi_{2}}\right)}+\frac{2}{\xi_{2}\left(2 \xi_{1}-\xi_{2}\right)}+\frac{1}{-\xi_{2}} B\left(\xi_{1}-\frac{d_{1}}{d_{2}} \xi_{2}\right) \\
& +\frac{1}{2 \xi_{1}-\xi_{2}} B\left(\frac{d_{1} \xi_{2}-d_{2} \xi_{1}}{d_{2}-2 d_{1}}\right) .
\end{aligned}
$$

The constant terms of $\mu^{L}\left(K_{i}\right)$ are Morelli's rational functions

$$
\begin{aligned}
& \mu_{0}^{L}\left(K_{0}\right)=\frac{d_{1}^{2}+3 d_{1} d_{2}+d_{2}^{2}}{12 d_{1} d_{2}}, \\
& \mu_{0}^{L}\left(K_{1}\right)=\frac{11 d_{1}^{2}-7 d_{1} d_{2}+d_{2}^{2}}{12 d_{1}\left(2 d_{1}-d_{2}\right)} \\
& \mu_{0}^{L}\left(K_{2}\right)=\frac{d_{1}^{2}-4 d_{1} d_{2}+2 d_{2}^{2}}{-6 d_{2}\left(2 d_{1}-d_{2}\right)}
\end{aligned}
$$

agreeing with the numbers shown in Figure 2 in the introduction.

Again, one can verify by direct computation that $\sum_{i=0}^{2} \mu_{0}^{L}\left(K_{i}\right)=1$ and

$$
\sum_{F} \mu^{L}(\operatorname{Supp}(P, F)) I(F)=S(P)=1+e^{\xi_{1}}+e^{2 \xi_{1}}+e^{\xi_{2}} .
$$

Example 5.9. We consider the same triangle $P$ from Example 5.8, but now with a complement map $\Psi$ coming from an inner product $Q$ on $V^{*}$, given by the matrix

$$
\left(\begin{array}{ll}
a & b \\
b & c
\end{array}\right)
$$

As in Example 5.8, the function $\mu^{Q}$ of the singular cone $K_{2}$ may be computed either by subdividing, or by direct use of the defining property of interpolators. We find that $\mu^{Q}\left(K_{2}\right)$ is given by

$$
\begin{array}{r}
\frac{1+e^{\xi_{1}-\xi_{2}}}{\left(1-e^{-\xi_{2}}\right)\left(1-e^{2 \xi_{1}-\xi_{2}}\right)}+\frac{2}{\xi_{2}\left(2 \xi_{1}-\xi_{2}\right)}+\frac{1}{-\xi_{2}} B\left(\xi_{1}-\frac{b}{a} \xi_{2}\right) \\
+\frac{1}{2 \xi_{1}-\xi_{2}} B\left(\frac{-a \xi_{1}-2 b \xi_{1}-b \xi_{2}-2 c \xi_{2}}{a+4 b+4 c}\right) .
\end{array}
$$

The constant terms, in agreement with the construction of $[\mathrm{PT}]$, are

$$
\begin{aligned}
\mu_{0}^{Q}\left(K_{0}\right) & =\frac{3 a c-a b-b c}{12 a c}, \\
\mu_{0}^{Q}\left(K_{1}\right) & =\frac{a b+5 a c+4 b^{2}+25 b c+22 c^{2}}{12\left(a c+4 b c+4 c^{2}\right)}, \\
\mu_{0}^{Q}\left(K_{2}\right) & =\frac{2 a^{2}+8 a b+7 a c+2 b^{2}+2 b c}{6\left(a^{2}+4 a b+4 b c\right)},
\end{aligned}
$$

which add up to 1 . For the standard inner product, these coefficients are

$$
\mu_{0}^{Q}\left(K_{0}\right)=\frac{1}{4}, \quad \mu_{0}^{Q}\left(K_{1}\right)=\frac{9}{20}, \quad \mu_{0}^{Q}\left(K_{2}\right)=\frac{3}{10} .
$$


Again for comparison, let us detail how these numbers arise out of the construction of Pommersheim-Thomas. To apply [PT, Cor.1(iv)], we first consider the outer normal fan of $P$. The rays of this fan are $\rho_{0}=(1,2), \rho_{1}=(-1,0)$, and $\rho_{2}=(0,-1)$. A non-singular subdivision is achieved by adding the ray $\rho_{3}=(0,1)$. As before, we multiply the Todd polynomial $T=\sum_{i<j} D_{i} D_{j}+\frac{1}{12} \sum_{i} D_{i}^{2}$ in the ring given in [PT, Proposition 2]. One calculates $D_{0}^{2}=\frac{1}{5}\left(2 D_{0} D_{2}-2 D_{0} D_{3}\right), D_{1}^{2}=0, D_{2}^{2}=2 D_{0} D_{2}$, and $D_{3}^{2}=-2 D_{0} D_{3}$. Hence

$$
T=\frac{1}{4} D_{1} D_{2}+\frac{9}{20} D_{0} D_{2}+\frac{1}{20} D_{0} D_{3}+\frac{1}{4} D_{1} D_{3} .
$$

Using additivity under subdivision [PT, Cor.1(iii)], one finds $\mu_{0}\left(K_{0}\right)=\frac{1}{4}, \mu_{0}\left(K_{1}\right)=$ $\frac{9}{20}$, and $\mu_{0}\left(K_{2}\right)=\frac{1}{20}+\frac{1}{4}=\frac{3}{10}$.

\section{Comparison with Morelli's work}

In this section we compute values of $\nu$ and discuss the relations Morelli noticed between the constant terms of $\mu$ and $\nu$.

6.1. Computation of $\nu^{\Psi}$ for low-dimensional cones. For cones of dimension 0,1 and 2 it is easy to compute $\nu^{\Psi}(K)$ explicitly, just as we did for $\mu^{\Psi}(K)$ in Section 5, The proofs in this case are similar.

Proposition 6.1. Suppose that $K=\{0\}$ in the vector space $V=\{0\}$. Then $\nu(K)$ is the constant function 1 , independent of $\Psi$.

Proposition 6.2. Now suppose that $V$ is 1 -dimensional and $K=\operatorname{Cone}(v)$ is a ray in $V$ generated by a primitive vector $v \in \Lambda$. Then, independent of $\Psi$, we have

$$
\nu(K)(\xi)=B(-\langle\xi, v\rangle) .
$$

Proposition 6.3. With the notation of Proposition 5.3, we have

$$
\nu^{L}(K)(\xi)=B\left(-\frac{\langle\xi, c\rangle}{\langle\rho, c\rangle}\right)
$$

and

$$
\nu^{Q}(K)(\xi)=B\left(-\frac{Q(\xi, \rho)}{Q(\rho, \rho)}\right) .
$$

Proposition 6.4. With the notation of Proposition [5.4, we have

$$
\begin{aligned}
\nu^{L}(K)=\frac{1}{\left\langle\xi, v_{1}\right\rangle\left\langle\xi, v_{2}\right\rangle}- & {\left[\frac{e^{\left\langle\xi, v_{1}\right\rangle}}{1-e^{\left\langle\xi, v_{1}\right\rangle} B\left(-\frac{\langle\xi, c\rangle}{\left\langle\rho_{1}, c\right\rangle}\right)+}+\frac{e^{\left\langle\xi, v_{2}\right\rangle}}{1-e^{\left\langle\xi, v_{2}\right\rangle} B\left(-\frac{\langle\xi, c\rangle}{\left\langle\rho_{2}, c\right\rangle}\right)}\right.} \\
& \left.+\frac{e^{\left\langle\xi, v_{1}\right\rangle} e^{\left\langle\xi, v_{2}\right\rangle}}{\left(1-e^{\left\langle\xi, v_{1}\right\rangle}\right)\left(1-e^{\left\langle\xi, v_{2}\right\rangle}\right)}\right]
\end{aligned}
$$

and

$$
\begin{aligned}
\nu^{Q}(K)=\frac{1}{\left\langle\xi, v_{1}\right\rangle\left\langle\xi, v_{2}\right\rangle}- & {\left[\frac{e^{\left\langle\xi, v_{1}\right\rangle}}{1-e^{\left\langle\xi, v_{1}\right\rangle}} B\left(-\frac{Q\left(\xi, \rho_{1}\right)}{Q\left(\rho_{1}, \rho_{1}\right)}\right)+\frac{e^{\left\langle\xi, v_{2}\right\rangle}}{1-e^{\left\langle\xi, v_{2}\right\rangle} B\left(-\frac{Q\left(\xi, \rho_{2}\right)}{Q\left(\rho_{2}, \rho_{2}\right)}\right)}\right.} \\
& \left.+\frac{e^{\left\langle\xi, v_{1}\right\rangle} e^{\left\langle\xi, v_{2}\right\rangle}}{\left(1-e^{\left\langle\xi, v_{1}\right\rangle}\right)\left(1-e^{\left\langle\xi, v_{2}\right\rangle}\right)}\right] .
\end{aligned}
$$


6.2. Morelli's coincidence in dimensions $\mathbf{1}$ and 2 . In this section we will prove Morelli's observation (24) for lattice cones of dimension at most 2, using the computations of $\nu^{\Psi}(K)$ for cones $K$ of dimension at most 2 from Section 6.1 ] Using these propositions, we can prove the following relation between $\mu$ and $\nu$ for cones of dimension at most 2. The following theorem, proved by Morelli in the flag case, shows that the coincidence of constant terms holds also in the case of inner products.

Theorem 8. Let $K$ be a cone with vertex at 0 in $V$, and assume that the dimension of $V$ is at most 2. Let $\Psi$ be a complement map on $V$ induced by an inner product on $V^{*}$ or a complete flag in $V^{*}$ and let $\Psi^{*}$ be the corresponding complement map on $V$. Then we have

$$
\nu^{\Psi}(K)(0)=\mu^{\Psi^{*}}\left(K^{\vee}\right)(0) .
$$

Proof. For cones of dimension 0 , the constant term of both $\mu$ and $\nu$ is always equal to 1 . For cones of dimension 1 , the constant term of both $\mu$ and $\nu$ is always $1 / 2$. Now let $K$ have dimension 2. From the inversion formula of Theorem 7 , it follows that

$$
\mu(K)(0)+\nu(K)(0)=\frac{1}{2}
$$

Hence, the desired conclusion is equivalent to

$$
\mu(K)(0)+\mu\left(K^{\vee}\right)(0)=\frac{1}{2} .
$$

Suppose that $\mu$ is induced by an inner product. The inner product gives us an identification of $V$ with $V^{*}$, allowing us to consider $K$ and $K^{\vee}$ as both living in the same space $V$. If $K=\operatorname{Cone}\left(v_{1}, v_{2}\right)$, then $K^{\vee}=\operatorname{Cone}\left(w_{1}, w_{2}\right)$ with $v_{i}$ orthogonal to $w_{i}, i=1,2$, under the inner product. Furthermore, considering the orthogonal cones $L_{1}=\operatorname{Cone}\left(v_{1}, w_{1}\right), L_{2}=\operatorname{Cone}\left(v_{2}, w_{2}\right)$, we have the following equation of characteristic functions:

$$
\chi_{K_{1}}+\chi_{K^{\vee}}=\chi_{L_{1}}+\chi_{L_{2}}
$$

and hence

$$
\mu\left(K_{1}\right)+\mu\left(K^{\vee}\right)=\mu\left(L_{1}\right)+\mu\left(L_{2}\right) .
$$

Now the desired result follows from the fact that $\mu(L)(0)=1 / 4$ for orthogonal 2dimensional cones $L$. To see this, note that $L$ and its image $\operatorname{rot}_{\pi / 2}(L)$ under rotation by $\pi / 2$ form a half-space, and rotation is an isomorphism of $V$ that preserves the inner product; hence $\mu(L)(0)=\mu\left(\operatorname{rot}_{\pi / 2}(L)\right)(0)$.

Now suppose that $\Psi$ is induced by a complete flag in $V^{*}$. This flag is determined by a line $U$ in $V^{*}$, and $\Psi^{*}$ is induced by the dual flag, determined by the dual line $U^{*}$ in $V$. Now choose an identification $i: V \rightarrow V^{*}$. Under this identification $U$ pulls back to a line perpendicular to $U^{*}$. It follows that

$$
\mu^{\Psi^{*}}\left(K^{\vee}\right)(0)=\mu^{\Psi}\left(\operatorname{rot}_{\pi / 2} i^{-1}\left(K^{\vee}\right)\right)(0) .
$$

But $\operatorname{rot}_{\pi / 2} i^{-1}\left(K^{\vee}\right)$ and $K$ fit together to form a half-space. Hence

$$
\mu^{\Psi}(K)(0)+\mu^{\Psi}\left(\operatorname{rot}_{\pi / 2} i^{-1}\left(K^{\vee}\right)\right)(0)=\frac{1}{2} .
$$

The result follows. 
Example 6.5. Consider the triangle $P$ from Example 5.6 with vertices $v_{0}=$ $(0,0), v_{1}=(1,0), v_{2}=(0,1)$. For $\Psi$ corresponding to the standard inner product, recall that the constant terms of the $\mu$ of the vertex cones are $1 / 2,3 / 8$, and $3 / 8$. According to the discussion above, we have that the constant terms of the $\nu$ 's are found by subtracting from $1 / 2$ :

$$
\nu\left(K_{0}\right)(0)=\frac{1}{4}, \quad \nu\left(K_{1}\right)(0)=\frac{1}{8}, \quad \nu\left(K_{2}\right)(0)=\frac{1}{8} .
$$

One can then check that the volume of this triangle is given by summing $\nu(F)(0)$ values times the number lattice points in the relative interior of $F$. Indeed, since the only lattice points are the vertices, one has

$$
\operatorname{Vol}(P)=\frac{1}{4}+\frac{1}{8}+\frac{1}{8}=\frac{1}{2} .
$$

For the triangle from Example 5.8, with vertices $v_{0}=(0,0), v_{1}=(2,0), v_{2}=$ $(0,1)$, one similarly computes the $\nu(K)$ constant terms (again using the standard inner product):

$$
\nu\left(K_{0}\right)(0)=\frac{1}{4}, \quad \nu\left(K_{1}\right)(0)=\frac{1}{20}, \quad \nu\left(K_{2}\right)(0)=\frac{1}{5} .
$$

This time there is one lattice point in the relative interior of an edge. The corresponding $\nu$ constant term is $1 / 2$, according to Proposition 6.3. Thus, we check that

$$
\operatorname{Vol}(P)=\frac{1}{4}+\frac{1}{20}+\frac{1}{5}+\frac{1}{2}=1 .
$$

\section{REFERENCES}

[Bv] A. Barvinok, A polynomial time algorithm for counting integral points in polyhedra when the dimension is fixed, Math. Oper. Res. 19 (1994) 769-779. MR.1304623 (96c:52026)

[BP] A. Barvinok and J.E. Pommersheim, An algorithmic theory of lattice points in polyhedra, Math. Sci. Res. Inst. Publ., 38 (1999) 91-147. MR.1731815 (2000k:52014)

[BV] N. Berline and M. Vergne, Local Euler-Maclaurin formula for polytopes, Mosc. Math. J. 7 (2007) 355-386. MR2343137 (2008k:52026)

[Br] M. Brion, Points entiers dans les polyèdres convexes, Ann. Sci. École Norm. Sup. (4) 21 (1988) 653-663. MR982338 (90d:52020)

[Fu] W. Fulton, Introduction to toric varieties. Annals of Mathematics Studies 131, Princeton University Press, 1993. MR 1234037 (94g:14028)

[La] J. Lawrence, Rational-function-valued valuations on polyhedra, DIMACS Ser. Discrete Math. Theoret. Comput. Sci.,6 (1991) 199-208. MR.1143297(92m:52025)

$[\mathrm{McM}]$ P. McMullen, Lattice invariant valuations on rational polytopes, Archiv Math. 31 (1978) 509-516. MR.526617 (80d:52011)

[Mo] R. Morelli, Pick's theorem and the Todd class of a toric variety, Adv. Math. 100 (1993) 183-231. MR1234309 (94j:14048)

[Pi] G.A. Pick, Geometrisches zur Zahlenlehre, Sitzenber. Lotos (Prague) 19 (1899) 311-319.

[PT] J. Pommersheim and H. Thomas, Cycles representing the Todd class of a toric variety, J. Amer. Math. Soc. 17 (2004) 983-994. MR2083474 (2005h:14124)

[Th] H. Thomas, Cycle-level intersection theory for toric varieties, Canad. J. Math. 56 (2004) 1094-1120. MR2085635(2005e:14083)

School of Mathematics, Georgia Institute of Technology, Atlanta, Georgia 30332 0160

E-mail address: stavros@math.gatech.edu

Department of Mathematics, Reed College, 3203 SE Woodstock Boulevard, PortLAND, OREGON 97202-8199

E-mail address: jamie@reed.edu 